\title{
Analysis of Amorphous and Oxide Surface Layers on Nanoparticles
}

\author{
Christopher R. Perrey, C. Barry Carter, James Bentley,* and Markus Lentzen** \\ Department of Chemical Engineering and Materials Science, University of Minnesota \\ 421 Washington Avenue S.E., Minneapolis, MN 55455-0132 \\ * Metals and Ceramics Division, Oak Ridge National Laboratory, PO Box 2008, Oak Ridge, TN \\ 37831-6064 \\ ** Institute for Solid State Research, Research Center Jülich GmbH, 52425 Jülich, Germany
}

As nanoparticles are considered for applications in mechanical and electrical devices, the surface quality of these nanoparticles has become of paramount importance. An example where surface structure and chemistry are important is the consideration of using individual Si nanoparticles for semiconductor devices [1]. If an insulating surface oxide layer is present, the difference in conductivity across the surface layer may be deleterious to the device. Similarly, oxide layers can affect the mechanical properties of single nanoparticles. For example, an oxide layer present on a $\mathrm{Si}$ nanosphere may prevent introduced dislocations from being ejected upon mechanical unloading, thus affecting plastic deformation, dislocation pile-up, and associated work hardening $[2,3]$.

This study focuses on the imaging and analysis of such surface layers using transmission electron microscopy (TEM). Different types of nanoparticles are considered, including $\mathrm{Si}$, SiC, and Ti. A representative $\mathrm{Si}$ nanosphere is seen in Figure 1. The spherical morphology of this particle is notable, as is the non-uniform surface layer present surrounding the particle. Information about this layer and its relationship to the crystalline nanoparticle, is provided by high-resolution TEM (HRTEM). However, with conventional HRTEM determination of the surface-film thickness is problematic, because the contrast from the film strongly overlaps that of the support film. The use of the $\mathrm{C}_{\mathrm{S}}$-corrected Philips CM200 FEG installed at Jülich provides a method improving this situation [4-7]: it minimizes the contrast of the support film by choosing an appropriate defocus. Then the particle rim is slightly out of focus, the amorphous film is in contrast, and its thickness can be measured undisturbed by any contrast from the support. Using HRTEM, this surface layer can be imaged as shown in Figure 2.

Further information about the structure and chemistry of the surface layers can be obtained using analytical TEM. A Philips CM200 FEG with Gatan Imaging Filter and Emispec Vision was used to produce spectral profiles across nanoparticles. Figure 3(a) shows an annular-dark-field scanning TEM (STEM) image of a Si nanoparticle with the trajectory of a drift-corrected STEM spectral profile noted [ 1 nA in $1.5 \mathrm{~nm}$ probe, 150 pixels, $1 \mathrm{~nm}$ spacing, $1 \mathrm{~s}$ dwell/pixel for core-loss electron energy-loss spectra (EELS)]. The spectra confirm the presence of an oxide surface layer on the $\mathrm{Si}$ nanoparticle. Figure 3(b) shows EELS data extracted from the spectral profile. The profile of the oxygen signal across the nanoparticle is as expected for a spherical nanoparticle composed of a $\mathrm{Si}$ "core" and a "shell" of an oxide layer on the particle surface. Using these methods, information can be obtained concerning the formation and subsequent oxidation of nanoscale surfaces.

\section{References}

[1] M.L. Ostraat, et al., Appl. Phys. Lett., 79 (3) (2001) 433.

[2] C.R. Perrey, et al., Mater. Res. Soc. Symp. Proc., 740 (2002), in press.

[3] W.W. Gerberich, et al., J. Mech. Phys. Solids, (2003), in press.

[4] M. Haider, et al., Nature, 392 (1998) 768.

[5] M. Haider, et al., Ultramicroscopy, 75 (1998) 53.

[6] M. Lentzen, et al., Ultramicroscopy, 92 (2002) 233. 
[7] C.L. Jia, M. Lentzen, and K. Urban, Science, 299 (2003) 870.

[8] This work was supported by the National Science Foundation under grant DMI-0103169. Prof. Knut Urban, Research Center Jülich, is also thanked for his encouragement and Dr. Lothar Houben for valuable discussions. The project on aberration-correction of a transmission electron microscope was funded by the Volkswagen Stifung. The authors gratefully acknowledge the members of the University of Minnesota's HPPD program, particularly A. Gidwani, R. Mukherjee, T. Renault, J. V. R. Heberlein, S. L. Girshick, and P. H. McMurry. Research at the ORNL SHaRE Collaborative Research Center was supported by the Division of Materials Sciences and Engineering, U.S. Department of Energy, under contract DE-AC0500OR22725 with UT-Battelle, LLC.

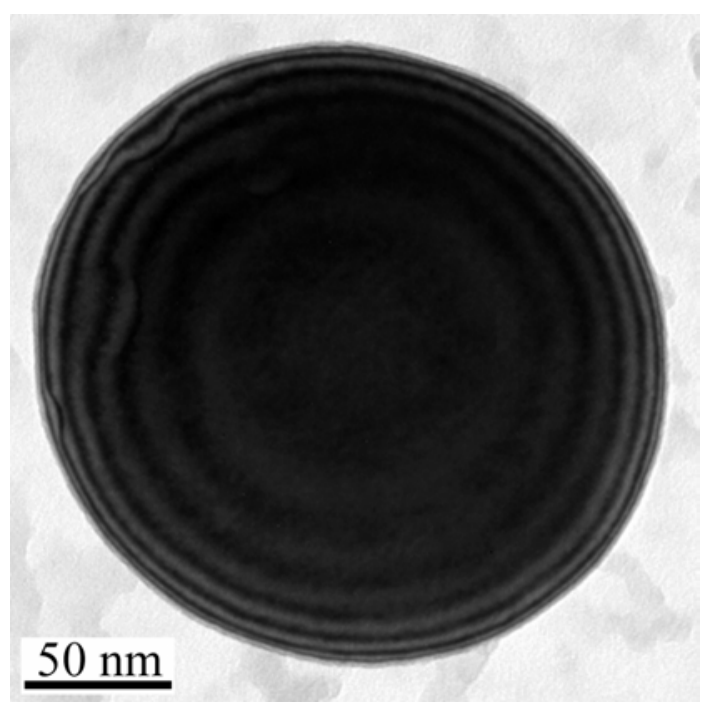

FIG. 1. Bright-field TEM image of a silicon nanoparticle.

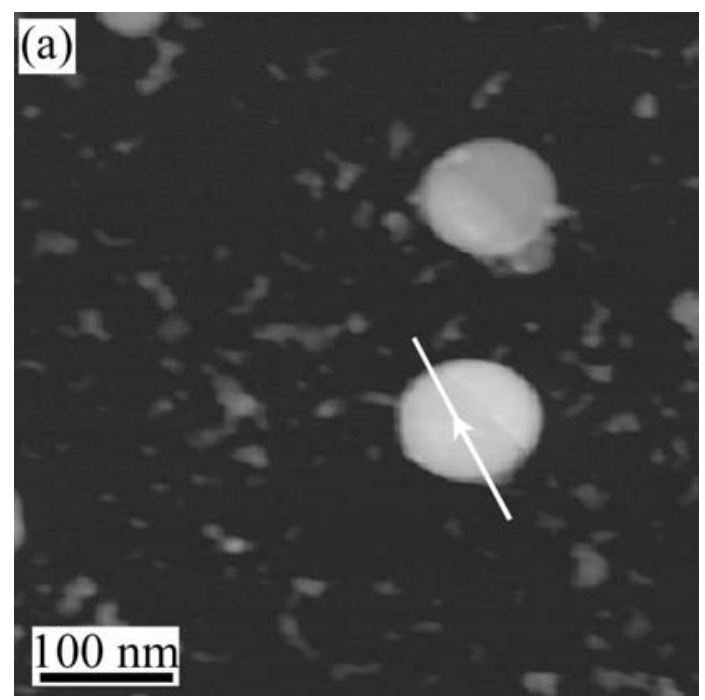

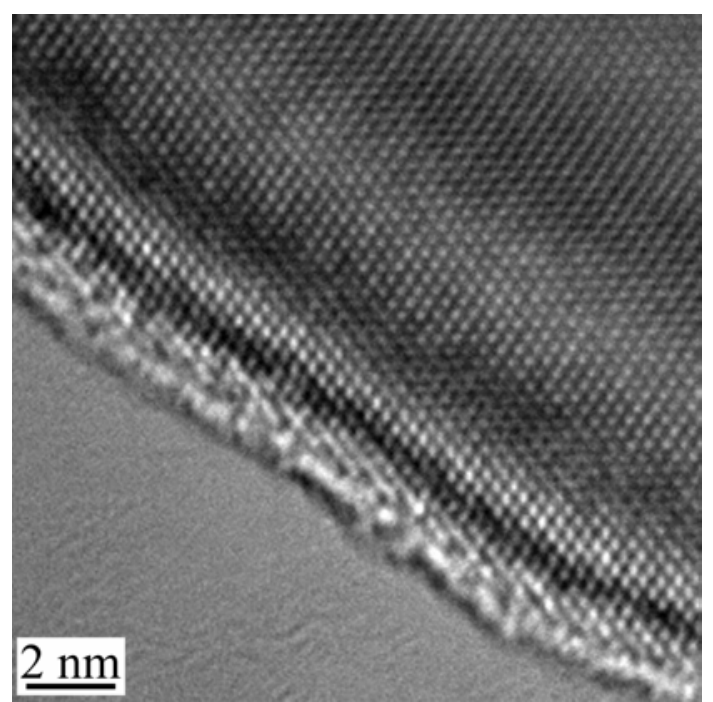

FIG. 2. HRTEM image of a Si nanosphere; the amorphous surface layer is visible.

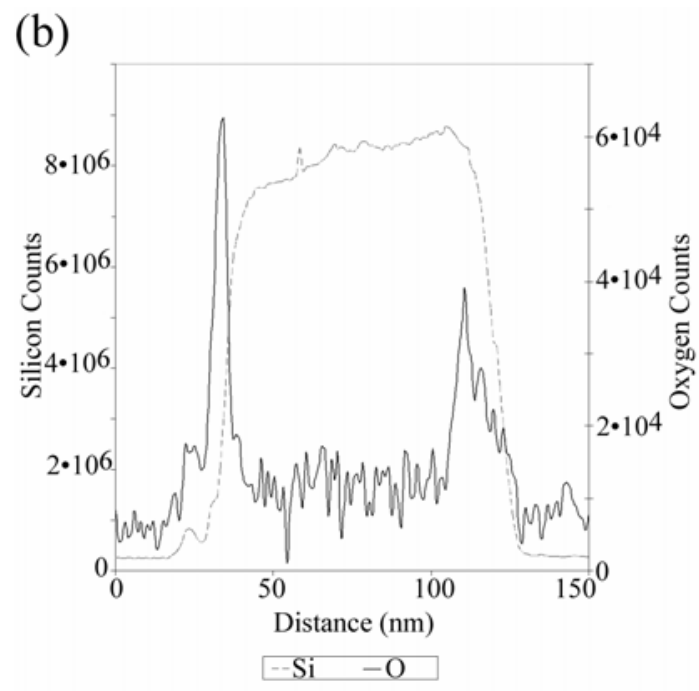

FIG. 3. The annular-dark-field STEM image in (a) designates the line trace for a spectral profile across a Si nanoparticle. The resulting PEELS oxygen and silicon signals are shown in (b) as a function of distance along the line scan. This profile is as expected for a particle that has a "core" of silicon and a "shell" oxide layer. 